\title{
Prognostic significance of galectin-I and vasculogenic mimicry in patients with gastric cancer
}

This article was published in the following Dove Press journal: OncoTargets and Therapy

\author{
Xiaolan You ${ }^{1,2}$ \\ Yuanjie Wang ${ }^{2}$ \\ Jian $\mathrm{Wu}^{2}$ \\ Qinghong $\mathrm{Liu}^{2}$ \\ Dehu Chen ${ }^{2}$ \\ Dong Tang ${ }^{3}$ \\ Daorong Wang ${ }^{3}$
}

'Department of Integrated Traditional Chinese and Western Medicine,

Medical College of Yangzhou

University, Yangzhou, Jiangsu

Province, People's Republic of China;

${ }^{2}$ Department of Gastrointestinal

Surgery, Taizhou People's Hospital,

Taizhou, Jiangsu Province, People's

Republic of China; ${ }^{3}$ Department of

Gastrointestinal Surgery, Clinical

Medical College of Yangzhou

University (Subei People's Hospital

of Jiangsu Province), Yangzhou, Jiangsu

Province, People's Republic of China
Correspondence: Daorong Wang

Department of Gastrointestinal Surgery, Clinical Medical College of Yangzhou University (Subei People's Hospital of jiangsu Province), 98\#, Nantong West Road, Yangzhou 225000, Jiangsu, People's Republic of China Email wdaorong666@sina.com
Introduction: We evaluated the expression of galectin-1 (Gal-1) and vasculogenic mimicry $(\mathrm{VM})$ in gastric cancer (GC) and investigated their relationships with the clinicopathological factors and prognostic significance in GC.

Materials and methods: Immunohistochemical (IHC) staining and CD34-periodic acid-Schiff double stain were used to investigate Gal-1 expression and VM in paraffin-embedded sections from 127 patients with GC of all tumor stages. The relationships between Gal-1 expression and VM, clinicopathological variables, and survival were analyzed. $P<0.05$ was considered statistically significant.

Results: Among the 127 cases, 86 (67.7\%) were positive for Gal-1; VM was detected in 29 cases (22.8\%). There was a significant association between VM and the Gal-1 IHC staining; all cases with VM were positive for Gal-1 staining. Gal-1 expression and VM in primary GC tissue were associated with tumor size, differentiation, depth of tumor invasion, stage, lymph node metastases, and tumor emboli in microvessels (all, $P<0.05$ ). Kaplan-Meier analysis revealed that the overall survival time was $52.56 \pm 2.44$ months ( $95 \%$ confidence interval [CI]: 47.77-57.35) for patients with Gal-1-negative and VM-negative primary GC tissue, $43.83 \pm 2.17$ months (95\% CI: 39.58-48.08) for patients with Gal-1-positive but VM-negative primary GC tissue, and $23.97 \pm 2.44$ months (95\% CI: 19.18-28.76) for patients with Gal-1-positive and VM-positive primary GC tissue $\left(\chi^{2}=\right.$ $60.21, P<0.01)$. Gal-1 expression was positively associated with VM in primary GC tissue.

Conclusion: Both Gal-1 expression and VM in primary GC tissue are indicators of poor prognosis for GC after gastrectomy, and Gal-1 may be a novel target for treating VM in GC.

Keywords: galectin-1, vasculogenic mimicry, gastric cancer, prognosis

\section{Introduction}

As one of the most common cancers worldwide, gastric cancer (GC) is the second leading cause of cancer-related mortality after lung cancer. ${ }^{1}$ Although a variety of treatments such as neoadjuvant chemotherapy and advanced surgical methods have improved the survival rate gradually over the past 30 years, the overall 5-year survival rate for resectable GC remains poor, especially that of the more advanced stages. ${ }^{2-4}$ Recently, treatment with ramucirumab, a monoclonal antibody that antagonizes VEGFR2, has become an important approach for treating GC. ${ }^{5}$ However, drugs targeting VEGF signaling have failed to improve the survival of patients in Phase II and III clinical trials. ${ }^{6}$ The presence of vasculogenic mimicry (VM) may represent a mechanism of resistance against angiostatic compounds. ${ }^{7,8}$

VM was first found in melanoma in $1999,{ }^{9}$ occurring when endothelium-dependent vessel growth is insufficient to support the rapid proliferation of tumor tissues, following which non-endothelial vascular networks provide oxygen and nutrients to tumors through a structure of channels, ${ }^{10-12}$ and it indicates that tumor cells can directly generate 
vascular channels that facilitate tumor perfusion independent of tumor angiogenesis by vascular endothelial cells. ${ }^{9,12}$ Maniotis et al defined this structure of channels composed of tumor basement membrane and tumor cells lacking blood vessel endothelium as VM. ${ }^{9}$ Based on these features, VM can be distinguished using immunohistochemical (IHC) staining and histochemical double staining. While VM is CD31- or CD34-negative (endothelial markers) and periodic acidSchiff(PAS)-positive, classic blood vessels are nevertheless double positive for endothelial and PAS markers. ${ }^{10,11}$ VM has been observed in many malignant tumors, including lung cancer, ${ }^{13}$ breast cancer, ${ }^{14}$ pancreatic cancer, ${ }^{15}$ glioblastoma, ${ }^{16}$ colorectal cancer, ${ }^{17}$ ovarian cancer, ${ }^{18}$ hepatocellular cancer, ${ }^{19}$ prostate cancer, ${ }^{20}$ esophageal cancer, ${ }^{21}$ and $\mathrm{GC},{ }^{22}$ and recent studies have shown that VM is associated with poor prognosis in human tumors. ${ }^{14,16,22} \mathrm{VM}$ can promote tumor growth and metastasis and is closely related to tumor neovascularization and cancer stem-like cells, which are associated with tumor invasion and drug resistance. ${ }^{14}$ However, few studies have explored VM as a potential prognostic marker and therapeutic target in GC, and the underlying molecular mechanisms of $\mathrm{VM}$ remain largely unknown.

Encoded by the LGALS1 gene, galectin-1 (Gal-1) is a $14-\mathrm{kDa}$ homodimer and prototype member of the galectin superfamily, which is characterized by high-affinity binding to $\beta$-galactosides through a well-conserved carbohydrate recognition domain. ${ }^{23}$ Increasing clinical evidence has confirmed that Gal-1 is involved in a variety of biological processes, including selective deletion of specific thymocytes during T-cell development, T-cell homeostasis, ${ }^{24}$ inflammatory responses, ${ }^{25}$ and fetomaternal tolerance. ${ }^{26}$ In addition, Gal-1 participates in tumor progression by evoking immunosuppression through the induction of activated T-cell apoptosis, transformation, angiogenesis, and metastasis and is associated with poor prognosis in many malignant tumors. ${ }^{27-30}$ A few studies have shown that Gal-1 overexpression in GC is associated with poor prognosis. ${ }^{30,31}$ Our previous studies have found that Gal-1 promotes epithelial-mesenchymal transition (EMT) in GC cells, ${ }^{32}$ and other studies have found that EMT is an important step in VM. ${ }^{33,34}$ However, no study has clarified the correlation between Gal-1 and VM in GC. In this study, we performed IHC staining to examine Gal-1, and IHC and histochemical double staining to examine VM in GC tissues. We aimed to determine whether Gal-1 expression levels and the presence of VM are correlated with each other and with GC clinicopathological features and prognosis, including survival.

\section{Materials and methods Patient information}

We enrolled 127 patients with gastric adenocarcinoma and complete clinicopathological and follow-up data in our study from July 2012 to May 2013. The patients were treated at the Department of Gastrointestinal Surgery, Taizhou People's Hospital, Taizhou, Jiangsu, People's Republic of China. Table 1 shows the patients' detailed clinicopathological data. All patients underwent radical gastrectomy for the primary tumor and D2 lymphadenectomy; no patient received chemotherapy or radiotherapy prior to surgery, had distant metastases prior to surgery, or other synchronous malignancies or serious diseases.

\section{Immunohistochemistry}

IHC staining of all specimens was performed on formalinfixed, paraffin-embedded tissue. Sections were cut at $4-\mu \mathrm{m}$ thickness. The sections were deparaffinized in xylene and rehydrated in gradient ethanol. Endogenous peroxidases were blocked with $3 \%$ hydrogen peroxide in methanol for $10 \mathrm{~min}$. The sections were washed with phosphate-buffered saline, and then pretreated with citrate buffer $(\mathrm{pH} \mathrm{6.0)}$ ) for $20 \mathrm{~min}$ at $95^{\circ} \mathrm{C}$ in a microwave oven for antigen retrieval. The slides were incubated with primary antibodies against Gal-1 (1:200; Cell Signaling Technology, Danvers, MA, USA) overnight at $4{ }^{\circ} \mathrm{C}$, followed by incubation with biotin-conjugated secondary antibodies, and then horseradish peroxidaseconjugated streptavidin. The sections were stained with diaminobenzidine (DAB), counterstained with hematoxylin, dehydrated, cleared, mounted, and coverslipped.

\section{CD34-PAS dual staining}

IHC staining was used to perform staining for CD34 (1:100; Abcam, Cambridge, UK). The procedure was the same as that described in the previous section. PAS staining was performed using a PAS staining kit (SN:DG0005; Leagene Biotechnology Co., Ltd., Beijing, People's Republic of China). After DAB reaction, the sections were treated with $0.5 \%$ periodic acid solution for $10 \mathrm{~min}$, and rinsed with distilled water three times for 3 min, followed by staining with Schiff solution for 20 min away from light. After rinsing with distilled water, the sections were counterstained with hematoxylin, dehydrated, cleared, mounted, and coverslipped.

\section{Evaluation of IHC staining and CD34-PAS dual staining}

Three sections per specimens were stained for each antibody. Two independent pathologists blinded to the patients' 
Table I Chi-square assessment of the associations between Gal-I IHC staining and VM and the clinicopathological features of 127 patients with GC

\begin{tabular}{|c|c|c|c|c|c|c|c|}
\hline \multirow[t]{2}{*}{ Parameters } & \multirow[t]{2}{*}{$\mathbf{n}$} & \multicolumn{2}{|l|}{ Gal-I } & \multirow[t]{2}{*}{$P$-value } & \multicolumn{2}{|l|}{ VM } & \multirow[t]{2}{*}{$P$-value } \\
\hline & & Negative & Positive & & Negative & Positive & \\
\hline Age (years) & & & & 0.391 & & & 0.099 \\
\hline$<60$ & 37 & 14 & 23 & & 25 & 12 & \\
\hline$\geq 60$ & 90 & 27 & 63 & & 73 & 17 & \\
\hline Sex & & & & 0.599 & & & 0.755 \\
\hline Male & 89 & 30 & 59 & & 68 & 21 & \\
\hline Female & 38 & 11 & 27 & & 30 & 8 & \\
\hline Tumor size $(\mathrm{cm})$ & & & & $<0.001$ & & & $<0.001$ \\
\hline$<2$ & 16 & 15 & 1 & & 16 & 0 & \\
\hline $2-5$ & 47 & 21 & 26 & & 46 & I & \\
\hline$>5$ & 64 & 5 & 59 & & 36 & 28 & \\
\hline Tumor location & & & & 0.758 & & & 0.136 \\
\hline Upper third & 12 & 5 & 7 & & 12 & 0 & \\
\hline Middle third & 65 & 20 & 45 & & 48 & 17 & \\
\hline Lower third & 50 & 16 & 34 & & 38 & 12 & \\
\hline Differentiation & & & & $<0.001$ & & & $<0.001$ \\
\hline Good & 15 & 14 & 1 & & 15 & 0 & \\
\hline Moderate & 72 & 21 & 51 & & 61 & 11 & \\
\hline Poor & 40 & 6 & 34 & & 22 & 18 & \\
\hline Depth of tumor invasion & & & & 0.002 & & & 0.025 \\
\hline TI & 15 & 10 & 5 & & 15 & 0 & \\
\hline $\mathrm{T} 2-\mathrm{T} 4$ & 112 & 31 & 81 & & 83 & 29 & \\
\hline TNM stage & & & & $<0.001$ & & & $<0.001$ \\
\hline I & 16 & 14 & 2 & & 16 & 0 & \\
\hline II & 39 & 20 & 19 & & 38 & I & \\
\hline III & 61 & 4 & 57 & & 41 & 20 & \\
\hline IV & 11 & 3 & 8 & & 3 & 8 & \\
\hline Lymph node status & & & & $<0.001$ & & & $<0.001$ \\
\hline Positive & 71 & 7 & 64 & & 45 & 26 & \\
\hline Negative & 56 & 34 & 22 & & 53 & 3 & \\
\hline $\begin{array}{l}\text { Tumor emboli in the } \\
\text { microvessels }\end{array}$ & & & & $<0.00$ I & & & 0.016 \\
\hline Positive & 44 & 4 & 40 & & 20 & 24 & \\
\hline Negative & 83 & 37 & 46 & & 78 & 5 & \\
\hline
\end{tabular}

Abbreviations: Gal-I, galectin-I; IHC, immunohistochemical; VM, vasculogenic mimicry; GC, gastric cancer.

clinical status assessed the results. Negative controls, from which primary antibodies had been omitted, were treated identically; positive controls were provided by the kit supplier. To quantify Gal-1 immunostaining, the slides were imaged digitally with equal light exposure and evaluated with Image-Pro Plus, a digitalized IHC scoring program (Media Cybernetics, Rockville, MD, USA). The immunostaining was scored based on the product of the percentage of immunopositive cells $(0-100)$ multiplied by staining intensity score $(0,1,2$, and 3$)$ to yield scores of $0-300$. Receiver operating curve (ROC) statistics was employed to estimate cutoff points of the IHC score to distinguish high and low expression of Gal-1 in 127 GC samples. VM was defined as positive or negative. In CD34-PAS double staining, VM-positive status appeared as channel-like structures with negative CD34 and positive PAS staining, and containing red cells.

\section{Follow-up}

Patients underwent continuous follow-up up to July 30 , 2017. As patients without complete clinicopathological and follow-up data were excluded, no patient was lost to follow-up. The median follow-up duration after surgery was 39.6 months (range: $3.1-60.9$ months).

\section{Ethics}

The protocol of this trial was designed in accordance with the Declaration of Helsinki and was approved by the Clinical Research Ethics Committee of Taizhou People's Hospital 
(TZRY-EC-12-068). All patients were provided details on the assessment procedure, and all provided informed written consent.

\section{Statistical analysis}

Statistical analysis was conducted with SPSS 16.0 (SPSS, Chicago, IL, USA). Continuous variables are expressed as the means $\pm \mathrm{SE}$ and were compared between groups using Student's $t$-test. The correlations between Gal-1 expression and VM and the clinicopathological features were analyzed using the chi-square test. The Kaplan-Meier method was used for survival analysis; intergroup survival differences were assessed with log-rank testing. In all analyses, $P<0.05$ was considered statistically significant.

\section{Results}

\section{Gal-I expression and VM in GC tissues}

In the GC tissues, the median Gal-1 IHC scores were 78.29 (9.51-186.24). IHC staining of Gal-1 expression in GC tissues is shown in Figure 1. ROC statistics were used to estimate the IHC cutoff scores to distinguish positive and negative Gal-1 expression in the 127 cases. Scores $\geq 56.80$ were considered to indicate positive expression (Figure 2A). In accordance with this standard, Gal-1 expression was positive in 86 of $127 \mathrm{GC}$ cases $(67.7 \%)$ and negative in the remaining 41 cases $(32.3 \%)$. As Figure 2B shows, CD34PAS staining showed endogenous cell-dependent vessels (yellow arrow) and VM (green dotted line) in one GC specimen red blood cells are shown by green arrow in VM and endogenous cell-dependent vessels. CD34-PAS double

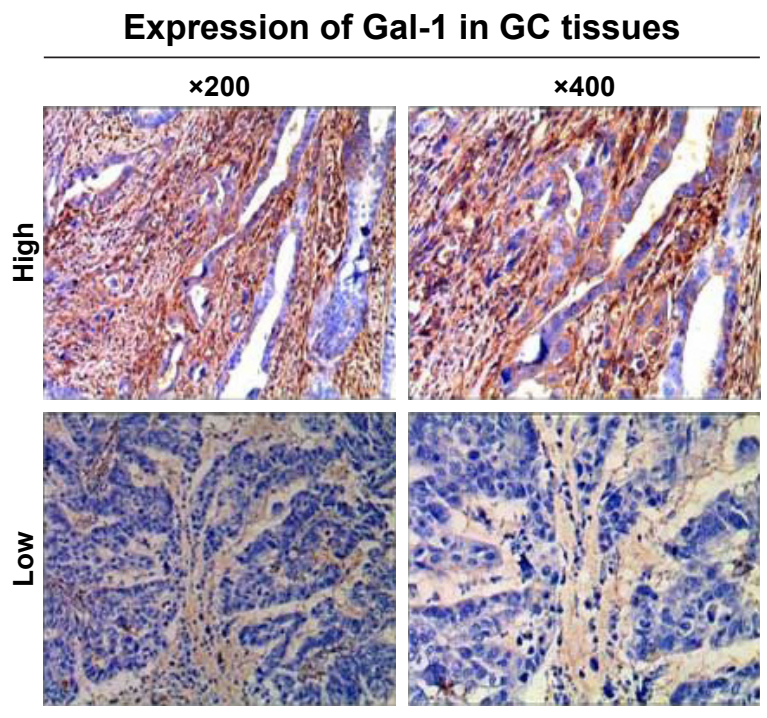

Figure I Immunohistochemical staining of Gal-I expression in GC tissues. Abbreviations: Gal-I, galectin-I; GC, gastric cancer. staining revealed VM in 29 cases $(22.8 \%)$; the remaining 98 cases were VM-negative (77.2\%).

\section{Correlation between Gal-I expression and VM and clinicopathological features}

The Gal-1 IHC scores in primary tumors with VM were significantly different from the Gal-1 score in primary tumors without $\mathrm{VM}(P<0.01$; Figure $3 \mathrm{~A})$, and there was a significant association between the Gal-1 IHC scores and VM $(r=0.669, P<0.01$; Figure 3B); all VM-positive samples were positive for Gal-1. Gal-1 expression and VM in primary GC tissue were associated with tumor size, differentiation, depth of tumor invasion, stage, lymph node metastases, and tumor emboli in the microvessels (all $P<0.05$ ), but were not correlated with age, sex, and tumor location (all $P>0.05$; Table 1).

\section{Correlation between Gal-I expression and $\mathrm{VM}$ and survival}

The final follow-up date was July 30, 2017. The median follow-up duration after surgery was 39.6 months (range: 3.1-60.9 months). A total of 68 patients (53.54\%) had tumor-related deaths. Kaplan-Meier analysis revealed that the overall survival (OS) time for Gal-1-positive and Gal-1negative patients was $52.56 \pm 2.44$ months ( $95 \%$ confidence interval [CI]: 47.77-57.35) and $37.17 \pm 1.95$ months $(95 \% \mathrm{CI}$ : 33.36-40.99), respectively. The OS rate of patients relative to Gal-1 expression status in GC tissue samples is shown in Figure 4A. Gal-1 overexpression was significantly associated with poor survival $\left(\chi^{2}=22.32, P<0.01\right)$. The OS time for VM-positive and VM-negative patients was $47.71 \pm 1.71$ months (95\% CI: 44.37-51.01) and $23.97 \pm 2.44$ months (95\% CI: 19.18-28.76), respectively. Additionally, the VMpositive patients had shorter OS time than the VM-negative patients. The OS rate of patients relative to VM status in GC tissue samples is shown in Figure 4B. The presence of VM was significantly associated with poor survival $\left(\chi^{2}=46.07\right.$, $P<0.01)$.

To evaluate the combined effect of Gal-1 expression and VM on GC prognosis, we classified the patients into three subgroups according to Gal-1 expression and VM in the primary GC tissue: Gal-1-negative and VM-negative, Gal-1-positive but VM-negative, and Gal-1-positive and VM-positive. Kaplan-Meier analysis revealed that OS time was $52.56 \pm 2.44$ months (95\% CI: 47.77-57.35) for Gal1-negative and VM-negative patients, $43.83 \pm 2.17$ months (95\% CI: 39.58-48.08) for Gal-1-positive but VM-negative patients, and $23.97 \pm 2.44$ months (95\% CI: 19.18-28.76) 

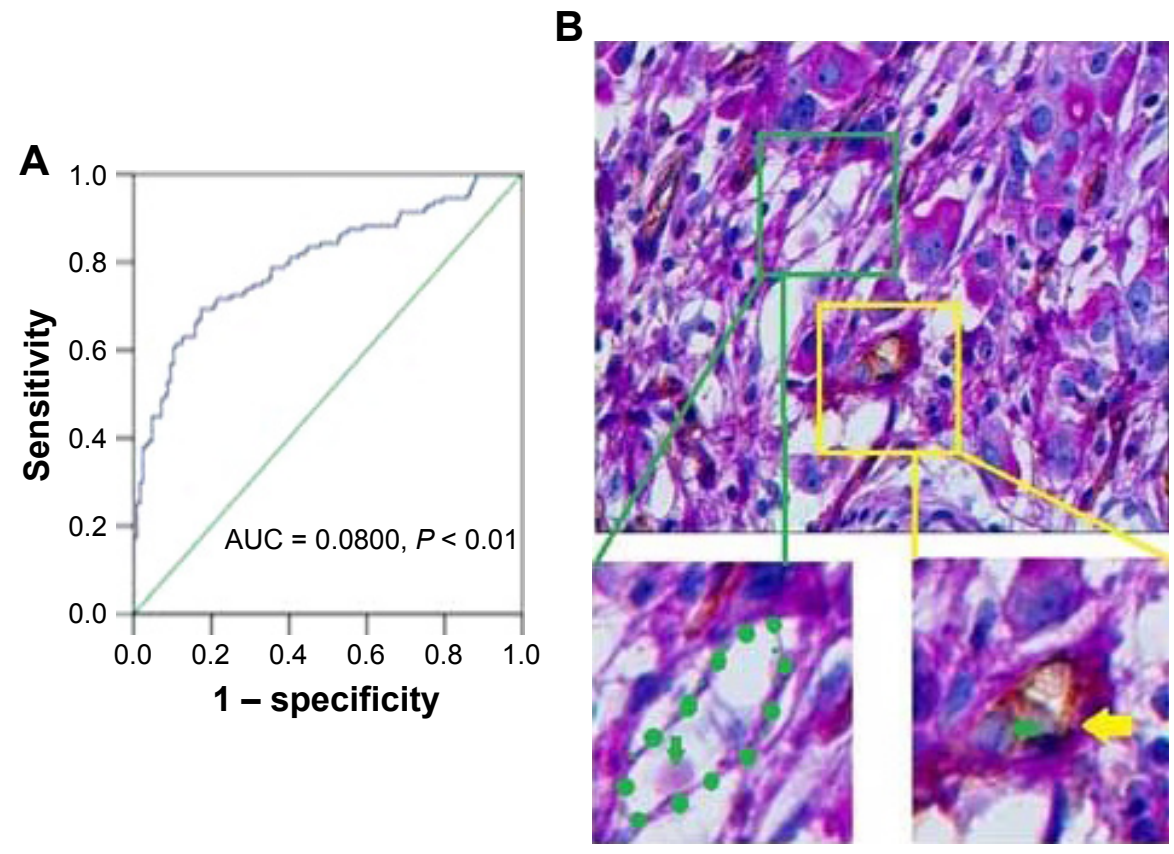

Figure 2 Gal-I expression and VM in GC tissues. (A) ROC statistics were used to estimate the Gal-I IHC cutoff score in human GC tissue. (B) CD34-PAS staining showing endogenous cell-dependent vessels (yellow arrow) and VM (green dotted line) in one GC specimen; red blood cells are shown by green arrow in VM and endogenous celldependent vessels. Original magnification: $\times 400$.

Abbreviations: Gal-I, galectin-I; VM, vasculogenic mimicry; GC, gastric cancer; ROC, receiver operating curve; IHC, immunohistochemical; PAS, periodic acid-Schiff; AUC, area under the curve.

for Gal-1-positive and VM-positive patients. The OS rates of groups with Gal-1-negative and VM-negative primary GC tissue, Gal-1-positive but VM-negative primary GC tissue, and Gal-1-positive and VM-positive primary GC tissue are shown in Figure 4C. Both Gal-1 overexpression and the presence of VM were significantly associated with poor survival $\left(\chi^{2}=60.21, P<0.01\right)$.

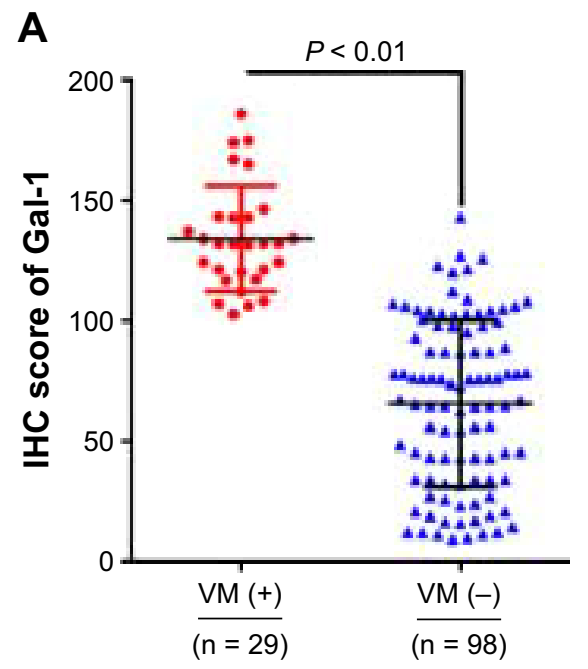

\section{Discussion}

Although efforts have been made regarding prevention, early diagnosis, and improved therapeutic strategies, the mortality rates for patients with advanced-stage GC remain high. ${ }^{1-4}$ Once patients develop resistance to chemotherapeutic regimens, antiangiogenesis therapy becomes an important approach for treating GC; endothelial-lined vessels are

B

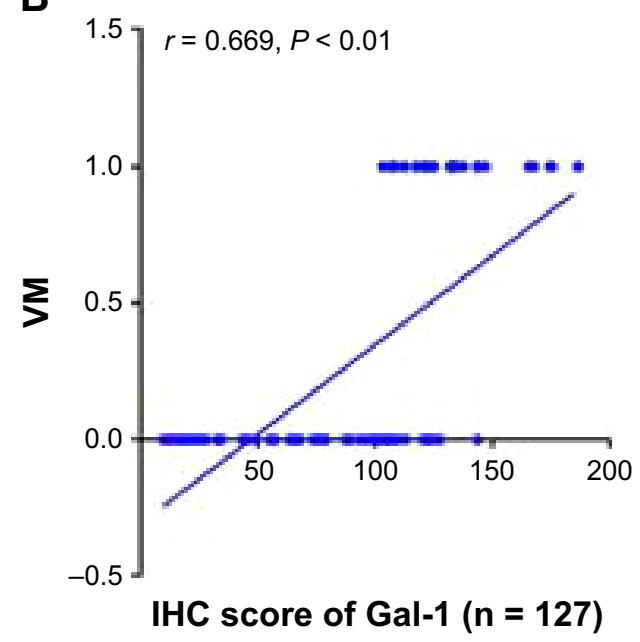

Figure 3 Correlation between Gal-I expression and VM and clinicopathological features. (A) The Gal-I IHC scores in primary tumors with VM were significantly different from Gal-I scores in primary tumors without VM $(P<0.0$ I). (B) Spearman correlation analysis showed significant correlation between Gal-I expression and VM $(r=0.669$, $P<0.00 \mathrm{I})$. The $Y$-axis values indicate VM status: 0, VM-negative; I, VM-positive.

Abbreviations: Gal-I, galectin-I; VM, vasculogenic mimicry; IHC, immunohistochemical. 
A

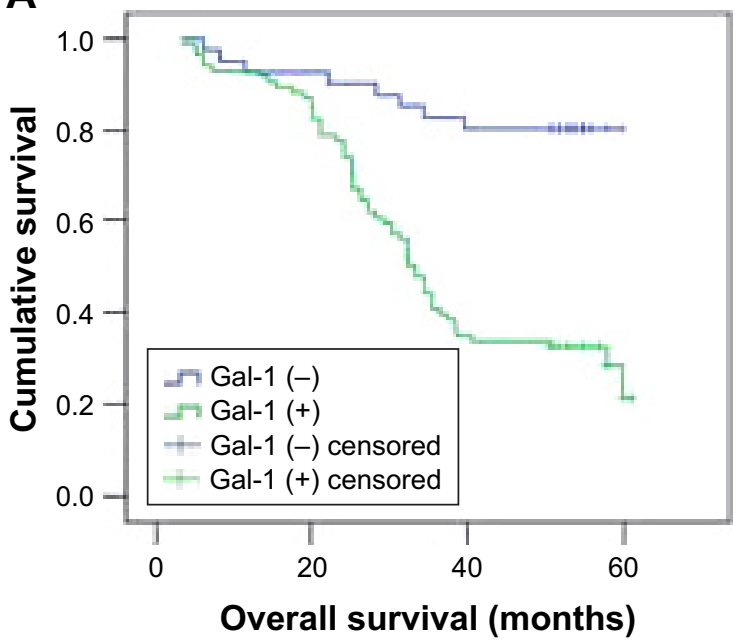

C

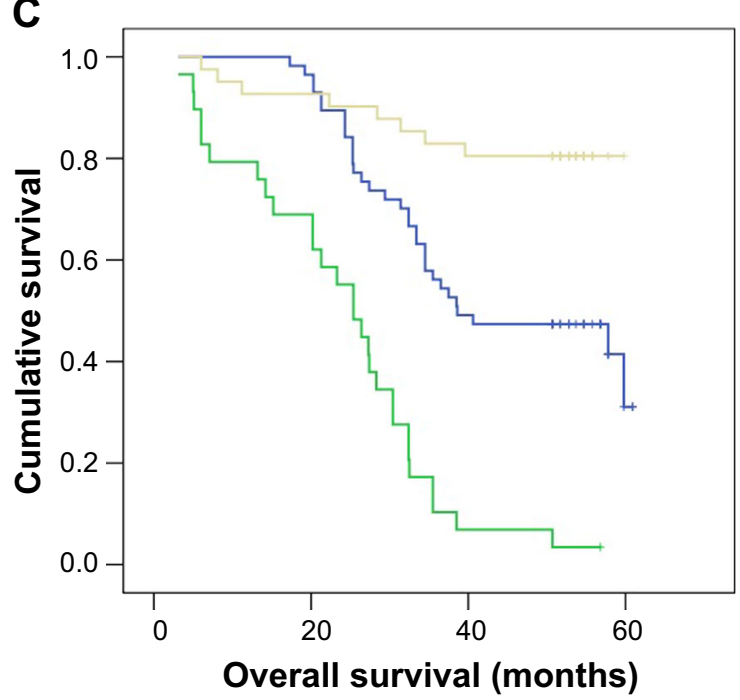

B

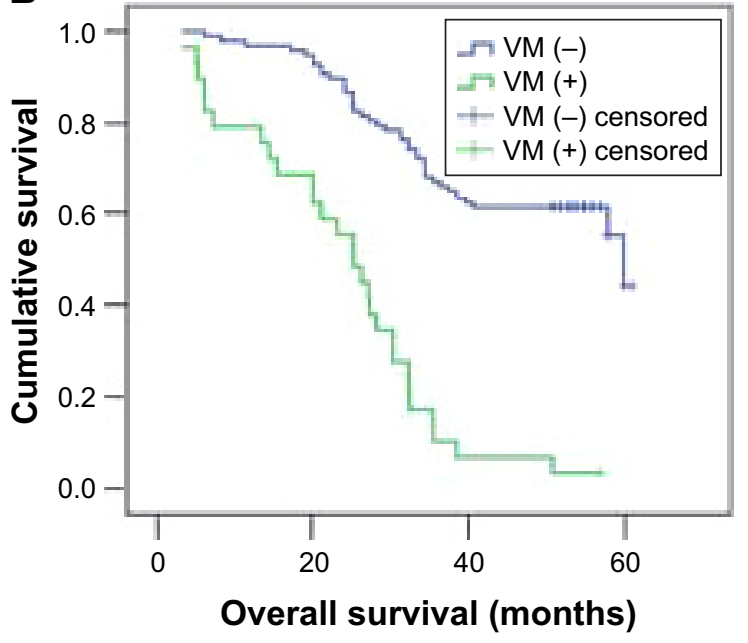

$\neg \mathrm{Gal}-1(+), \mathrm{VM}(-)$

$\neg \mathrm{Gal}-1(+), \mathrm{VM}(+)$

$\neg \mathrm{Gal}-1(-), \mathrm{VM}(-)$

+ Gal-1 (+), VM(-)-censored

+ Gal-1 (+), VM(+)-censored

Gal-1 (-), VM(-)-censored

Figure 4 OS rate of patients. (A) The OS rate of patients relative to Gal-I expression status in GC tissue samples. Gal-I overexpression was significantly associated with poor survival $(P<0.0 \mathrm{I})$. (B) The OS rate of patients relative to VM status in $\mathrm{GC}$ tissue samples. The presence of $\mathrm{VM}$ was significantly associated with poor survival $(P<0.0 \mathrm{I})$. (C) The OS rates of groups with Gal-I-negative and VM-negative primary GC tissue, Gal-I-positive but VM-negative primary GC tissue, and Gal-I-positive and VM-positive primary GC tissue. Both Gal-I overexpression and presence of $V M$ were significantly associated with poor survival $(P<0.0 \mathrm{I})$.

Abbreviations: OS, overall survival; Gal-I, galectin-I; GC, gastric cancer; VM, vasculogenic mimicry.

inhibited by some antiangiogenic agents initially, ${ }^{35}$ but the outcome of antiangiogenesis therapy is unsatisfactory, where VM and metastasis persistently increase thereafter. ${ }^{7}$ The occurrence of VM is thought to be a crucial step in GC progression and metastasis, ${ }^{22}$ suggesting that VM is a potential therapeutic target in GC and that VM-related molecules are potential targets for novel anti-GC therapies, and it is necessary to evaluate these new molecular targets for their potential roles as prognostic markers for patients with GC.

Gal-1 was the first protein discovered within the galectin family; it can form homodimers via non-covalent binding, which confers the ability to cross-link specific glycoconjugates. Intracellularly, Gal-1 is involved in pre-mRNA splicing; it interacts with oncogenic H-RAS and promotes cell migration, proving that it plays a key role in driving tumor transformation proteins to influence tumor progression, invasion, and angiogenesis. ${ }^{23}$ The literature largely reports that high Gal-1 levels correlate with tumor aggressiveness and the acquisition of a metastatic phenotype. ${ }^{27-30}$ LGALS1 amplification and overexpression have been demonstrated in thyroid, head and neck, colon, ovary, and prostate carcinoma, ${ }^{36}$ and high Gal-1 expression is associated with poor prognosis. ${ }^{27,28}$ However, as few studies have evaluated the correlation between Gal-1 overexpression and survival in $\mathrm{GC},{ }^{30}$ the prevalence of Gal-1 in GC and its relationship with prognosis remain largely unknown. In the present study, we examined $127 \mathrm{GC}$ samples for expression of the Gal-1 oncoprotein by immunohistochemistry. The median Gal-1 IHC score was 78.29 (9.51-186.24); in accordance with ROC statistics, 86 of $127 \mathrm{GC}$ cases had positive Gal-1 expression (67.7\%). 
Gal-1 expression was related to tumor size, differentiation, depth of tumor invasion, stage, lymph node metastases, and tumor emboli in the microvessels. Kaplan-Meier survival analysis confirmed a significant prognostic value of Gal-1 in GC. Gal-1-positive patients had significantly poorer outcome than Gal-1-negative patients. Thus, detecting Gal-1 expression in $\mathrm{GC}$ tissues might be helpful for prognosis in GC.

$\mathrm{VM}$ is found and considered a poor prognostic marker in many tumors; ${ }^{13-22} \mathrm{VM}$ provides essential nutrients to rapidly growing tumors to promote tumor growth, and based on the structure of VM less of endothelium, VM increases tumor perfusion by leaky vessels, and VM tubes may even connect with the endothelial-lined vasculature, ${ }^{10}$ which promotes tumor cell entry into the blood circulation, leading to metastasis. Drugs targeting endothelial signaling molecules such as bevacizumab, sunitinib, and sorafenib have been used clinically to treat various cancers, including GC, but their efficacy is limited. ${ }^{37}$ Some studies have found that targeted endothelial therapy may even contribute to $\mathrm{VM} \cdot{ }^{38}$ However, the clinical impact of VM on OS and prognosis in GC remains controversial. A study with insufficient clinical research indicated that VM is not closely associated with the prognosis of patients with GC. ${ }^{39}$ Here, we examined 127 GC cases for VM, where 29 (22.8\%) were VM-positive, and the incidence of VM increased with GC progression. VM was related to tumor size, differentiation, depth of tumor invasion, stage, lymph node metastases, and tumor emboli in the microvessels. We also confirmed a significant association between VM and poor survival.

The molecular mechanisms of VM are not fully understood; we show that Gal-1 IHC scores were significantly associated with VM in primary GC tissue, where all VMpositive samples were positive for Gal-1 staining. Gal-1 and VM had a concordant correlation with the clinicopathological features of GC, which suggests a connection between them. Our results also indicate that Gal-1 overexpression and the presence of VM, especially simultaneous Gal-1 overexpression and VM, are significantly correlated with poor survival in GC. Therefore, positivity for both Gal-1 and VM predicts a worse clinical outcome for patients with GC, and detecting Gal-1 expression and the presence of VM in primary GC tissue might be a novel prognostic marker.

Gal-1 is a direct target of HIF1, a key heterodimeric transcriptional factor for the cellular response to hypoxia. ${ }^{40}$ Gal-1 interacts with oncogenic H-RAS intracellularly, and RAS signaling can induce or enhance $\mathrm{SHH}$ expression, which can activate Hh signaling and promote the expression of Gli-1 and promote EMT of GC. ${ }^{32,41}$ EMT participates in VM formation, and as a main EMT-mediated process regulator, TWIST reportedly promotes the upregulation of VE-cadherin and VEGFR1 expression and contributes to VM formation. ${ }^{10}$ Therefore, we hypothesize that Gal-1 promotes VM in GC by regulating the RAS-Hh/Gli-1-TWIST signaling pathway. If this hypothesis is correct, Gal-1 and the pathway are potential therapeutic targets for GC. To date, the Gal-1 regulatory mechanism of VM in GC has not been well explored and requires further study.

\section{Conclusion}

Gal-1 expression is positively associated with VM in primary GC tissue. Both Gal-1 expression and the presence of VM in primary GC tissue are indicators of poor prognosis for GC after gastrectomy. Gal-1 may promote VM in GC by regulating the RAS-Hh/Gli-1-TWIST signaling pathway; Gal-1 and the pathway are potential therapeutic targets for GC.

\section{Acknowledgments}

This work was supported in part by China Postdoctoral Science Foundation (grant number 2018M632400), the National Natural Science Foundation of China (grant numbers 81172279 and 81572343). The authors would like to thank the native English-speaking scientists of Elixigen Company (Huntington Beach, CA, USA) for editing our manuscript.

\section{Disclosure}

The authors have no conflicts of interest to declare in this work.

\section{References}

1. Lin Y, Ueda J, Kikuchi S, et al. Comparative epidemiology of gastric cancer between Japan and China. World J Gastroenterol. 2011;17(39): $4421-4428$.

2. Zheng $\mathrm{L}$, Wu $\mathrm{C}, \mathrm{Xi} \mathrm{P}$, et al. The survival and the long-term trends of patients with gastric cancer in Shanghai, China. BMC Cancer. 2014; $14: 300$

3. Xu W, Beeharry MK, Liu W, Yan M, Zhu Z. Preoperative chemotherapy for gastric cancer: personal interventions and precision medicine Biomed Res Int. 2016;2016:3923585.

4. Son T, Hyung WJ. Laparoscopic gastric cancer surgery: current evidence and future perspectives. World J Gastroenterol. 2016;22(2):727-735.

5. Javle M, Smyth EC, Chau I. Ramucirumab: successfully targeting angiogenesis in gastric cancer. Clin Cancer Res. 2014;20(23):5875-5881.

6. Allegra CJ, Yothers G, O'Connell MJ, et al. Phase III trial assessing bevacizumab in stages II and III carcinoma of the colon: results of NSABP protocol C-08. J Clin Oncol. 2011;29(1):11-16.

7. Xu Y, Li Q, Li XY, Yang QY, Xu WW, Liu GL. Short-term anti-vascular endothelial growth factor treatment elicits vasculogenic mimicry formation of tumors to accelerate metastasis. J Exp Clin Cancer Res. 2012;31:16

8. Angara K, Borin TF, Arbab AS. Vascular mimicry: a novel neovascularization mechanism driving anti-angiogenic therapy (AAT) resistance in glioblastoma. Transl Oncol. 2017;10(4):650-660.

9. Maniotis AJ, Folberg R, Hess A, et al. Vascular channel formation by human melanoma cells in vivo and in vitro: vasculogenic mimicry. $A m J$ Pathol. 1999;155(3):739-752. 
10. Qiao L, Liang N, Zhang J, et al. Advanced research on vasculogenic mimicry in cancer. J Cell Mol Med. 2015;19(2):315-326.

11. Wang M, Zhao X, Zhu D, et al. HIF-1 $\alpha$ promoted vasculogenic mimicry formation in hepatocellular carcinoma through LOXL2 up-regulation in hypoxic tumor microenvironment. J Exp Clin Cancer Res. 2017; 36(1):60.

12. Racordon D, Valdivia A, Mingo G, et al. Structural and functional identification of vasculogenic mimicry in vitro. Sci Rep. 2017;7:6985.

13. Li Y, Sun B, Zhao X, et al. MMP-2 and MMP-13 affect vasculogenic mimicry formation in large cell lung cancer. $J$ Cell Mol Med. 2017;21(12):3741-3751.

14. Shen Y, Quan J, Wang M, et al. Tumor vasculogenic mimicry formation as an unfavorable prognostic indicator in patients with breast cancer. Oncotarget. 2017;8(34):56408-56416.

15. Yang J, Zhu DM, Zhou XG, et al. HIF- $2 \alpha$ promotes the formation of vasculogenic mimicry in pancreatic cancer by regulating the binding of Twist1 to the VE-cadherin promoter. Oncotarget. 2017;8(29): 47801-47815.

16. Han G, Li Y, Cao Y, et al. Overexpression of leptin receptor in human glioblastoma: correlation with vasculogenic mimicry and poor prognosis. Oncotarget. 2017;8(35):58163-58171.

17. Li W, Zong S, Shi Q, Li H, Xu J, Hou F. Hypoxia-induced vasculogenic mimicry formation in human colorectal cancer cells: involvement of HIF-1a, Claudin-4, and E-cadherin and Vimentin. Sci Rep. 2016;6:37534.

18. Tang J, Wang J, Fan L, et al. cRGD inhibits vasculogenic mimicry formation by down-regulating uPA expression and reducing EMT in ovarian cancer. Oncotarget. 2016;7(17):24050-24062.

19. Zhao N, Sun BC, Zhao XL, et al. Role of Bcl-2 and its associated miRNAs in vasculogenic mimicry of hepatocellular carcinoma. Int $J$ Clin Exp Pathol. 2015;8(12):15759-15768.

20. Wang $\mathrm{H}$, Lin $\mathrm{H}$, Pan J, et al. Vasculogenic mimicry in prostate cancer: the roles of EphA2 and PI3K. J Cancer. 2016;7(9):1114-1124.

21. Tang NN, Zhu H, Zhang HJ, et al. HIF- $1 \alpha$ induces VE-cadherin expression and modulates vasculogenic mimicry in esophageal carcinoma cells. World J Gastroenterol. 2014;20(47):17894-17904.

22. Guo Q, Yuan Y, Jin Z, et al. Association between tumor vasculogenic mimicry and the poor prognosis of gastric cancer in China: an updated systematic review and meta-analysis. Biomed Res Int. 2016;2016: 2408645.

23. Barondes SH, Castronovo V, Cooper DN, et al. Galectins: a family of animal beta-galactoside-binding lectins. Cell. 1994;76(4):597-598.

24. Sotomayor CE, Rabinovich GA. Galectin-1 induces central and peripheral cell death: implications in T-cell physiopathology. Dev Immunol. 2000;7(2-4):117-129.

25. Al-Salam S, Hashmi S. Galectin-1 in early acute myocardial infarction. PLoS One. 2014;9(1):e86994.

26. Than NG, Romero R, Erez O, et al. Emergence of hormonal and redox regulation of galectin-1 in placental mammals: implication in maternalfetal immune tolerance. Proc NatI Acad Sci U S A. 2008;105(41): 15819-15824.
27. Chen L, Yao Y, Sun L, et al. Clinical implication of the serum galectin-1 expression in epithelial ovarian cancer patients. J Ovarian Res. 2015;8:78.

28. Su YC, Davuluri GV, Chen CH, et al. Galectin-1-induced autophagy facilitates cisplatin resistance of hepatocellular carcinoma. PLoS One. 2016;11(2): $\mathrm{e} 0148408$

29. Jouve N, Despoix N, Espeli M, et al. The involvement of CD146 and its novel ligand galectin-1 in apoptotic regulation of endothelial cells. J Biol Chem. 2013;288(4):2571-2579.

30. He XJ, Tao HQ, Hu ZM, et al. Expression of galectin-1 in carcinomaassociated fibroblasts promotes gastric cancer cell invasion through upregulation of integrin $\beta 1$. Cancer Sci. 2014;105(11):1402-1410.

31. Chen J, Zhou SJ, Zhang Y, et al. Clinicopathological and prognostic significance of galectin-1 and vascular endothelial growth factor expression in gastric cancer. World J Gastroenterol. 2013;19(13): 2073-2079.

32. Chong Y, Tang D, Gao J, et al. Galectin-1 induces invasion and the epithelial-mesenchymal transition in human gastric cancer cells via noncanonical activation of the hedgehog signaling pathway. Oncotarget. 2016;7(50):83611-83626.

33. Liu Q, Qiao L, Liang N, et al. The relationship between vasculogenic mimicry and epithelial-mesenchymal transitions. J Cell Mol Med. 2016;20(9):1761-1769.

34. Han C, Sun B, Zhao X, et al. Phosphorylation of STAT3 promotes vasculogenic mimicry by inducing epithelial-to-mesenchymal transition in colorectal cancer. Technol Cancer Res Treat. 2017;16(6): 1209-1219.

35. Chen LT, Oh DY, Ryu MH, et al. Anti-angiogenic therapy in patients with advanced gastric and gastroesophageal junction cancer: a systematic review. Cancer Res Treat. 2017;49(4):851-868.

36. Cousin JM, Cloninger MJ. The role of galectin-1 in cancer progression, and synthetic multivalent systems for the study of galectin-1. Int $J$ Mol Sci. 2016;17(9):pii:E1566.

37. Kreisl TN, Kim L, Moore K, et al. Phase II trial of single-agent bevacizumab followed by bevacizumab plus irinotecan at tumor progression in recurrent glioblastoma. J Clin Oncol. 2009;27(5):740-745.

38. Ebos JM, Lee CR, Cruz-Munoz W, Bjarnason GA, Christensen JG, Kerbel RS. Accelerated metastasis after short-term treatment with a potent inhibitor of tumor angiogenesis. Cancer Cell. 2009;15(3): 232-239.

39. Cao Z, Bao M, Miele L, Sarkar FH, Wang Z, Zhou Q. Tumour vasculogenic mimicry is associated with poor prognosis of human cancer patients: a systemic review and meta-analysis. Eur J Cancer. 2013;49(18): 3914-3923.

40. Zhao XY, Chen TT, Xia L, et al. Hypoxia inducible factor-1 mediates expression of galectin-1: the potential role in migration/invasion of colorectal cancer cells. Carcinogenesis. 2010;31(8):1367-1375.

41. Brechbiel J, Miller-Moslin K, Adjei AA. Crosstalk between hedgehog and other signaling pathways as a basis for combination therapies in cancer. Cancer Treat Rev. 2014;40(6):750-759.
OncoTargets and Therapy

\section{Publish your work in this journal}

OncoTargets and Therapy is an international, peer-reviewed, open access journal focusing on the pathological basis of all cancers, potential targets for therapy and treatment protocols employed to improve the management of cancer patients. The journal also focuses on the impact of management programs and new therapeutic agents and protocols on
Dovepress

patient perspectives such as quality of life, adherence and satisfaction. The manuscript management system is completely online and includes a very quick and fair peer-review system, which is all easy to use. Visit http://www.dovepress.com/testimonials.php to read real quotes from published authors. 